\title{
Exploring the impact of rotating rectangular plasmonic nano-hole arrays on the transmission spectra and its application as a plasmonic sensor
}

\author{
A. M. Mahros \\ amr.mahros@mena.vt.edu
}

\section{M. Tharwat}

I. Ashry

\begin{abstract}
Department of Physics, Faculty of Science, University of Jeddah, Jeddah 21432, Saudi Arabia Department of Engineering Mathematics and Physics, Faculty of Engineering, Alexandria University, Alexandria 21526, Egypt

Department of Electrical Engineering, Faculty of Engineering, King Abdulaziz University, Jeddah 21432, Saudi Arabia

Department of Engineering Mathematics and Physics, Faculty of Engineering, Alexandria University, Alexandria 21526, Egypt
\end{abstract}

Plasmonic nano-structures play a significant role in most recent photonic devices and applications. In this paper, we investigate the optical transmission spectra of rotatable periodic nano-metric apertures with different dimensions. This investigation includes monitoring the modification of both the transmission resonance wavelengths and peak transmittance at different dimensions and orientations of the nano-holes. The obtained results provide better insight to the interaction of light with periodic plasmonic nano-hole arrays. We find that nano-holes dimension/orientation can totally suppress an optical transmission, tune its resonance wavelengths, and change its peak values. Furthermore, we present the surface plasmonic resonance sensing as an application for the reported nano-hole array.

[DOI: http://dx.doi.org/10.2971/jeos.2015.15023]

Keywords: Computational electromagnetic methods, finite difference time domain, nano-hole array, optical transmission, surface plasmons

\section{INTRODUCTION}

Plasmonics have been recently employed in a myriad of significant applications such as, biomedical sensing [1], efficient solar cells [2], fluorescence microscopy [3], spontaneous emission engineering [4] and fabrication of nano-antennas [5]. Metallic films perforated with periodic nano-hole arrays have been considered as one of the most interesting plasmonic structures. Such plasmonic structures have received a significant amount of research attention because of their extraordinary optical transmission (EOT) [6]. Since the first discovery of their EOT [7], considerable theoretical and experimental efforts have been carried out about investigating their optical properties [8]-[10]. It was documented in the literature that the coupling / decoupling phenomenon between surface plasmons (SPs) of patterned metallic films and incident light results in appearance of multiple resonance peaks in their optical transmission spectra [6].

The EOT of patterned plasmonic structures can be modified through many physical and geometrical parameters including hole shape [8, 11, 12], size [13], and depth [14], film material [9], surrounding medium refractive index [15], and structural periodicity [10]. Furthermore, more tunability to the EOT was achieved by applying a magnetic field [16]. However, the ability of modifying the EOT spectral characteristics through rotating the nano-holes has not yet been thoroughly investigated in the literature. Different numerical methods are well suited to study the optical transmission spectra of thin metallic films perforated with nanohole arrays. In particular, Greens dynamics tensor model [17], finite element method [12], mul- tiple multipole technique [18], and finite difference time domain (FDTD) method [11, 15] have been employed in numerical analysis of plasmonic structures. FDTD method has been well established as a fast powerful tool for integrated and diffractive optics device simulations.

Here we use the 3D FDTD to investigate the optical transmission spectrum of rotatable plasmonic arrayed structure consisting of rectangular nano-holes inscribed in circles. This study includes monitoring the modification of both resonance wavelengths and peak transmittance due to rotating the rectangular nano-holes of different sizes. Furthermore, geometrical illustration to the electric field is provided to physically interpret the obtained results. The results presented here should be useful for a large number of nano-structured photonic applications such as surface plasmonic resonance sensing.

\section{STRUCTURE DESCRIPTION AND FDTD SIMULATION}

In this work, optical transmission spectra of the reported plasmonic arrays are obtained using the FDTD algorithm which is trustworthy in solving Maxwells equations for different materials. The FDTD method is applied by using the OptiFDTD simulation tool from Optiwave Inc. The designed plasmonic structure layout is a nano-hole array perforated in a gold thin film "sandwiched" between glass substrate and air cladding as shown in Figure 1(a). The nano-holes have a rectangular 
(a)

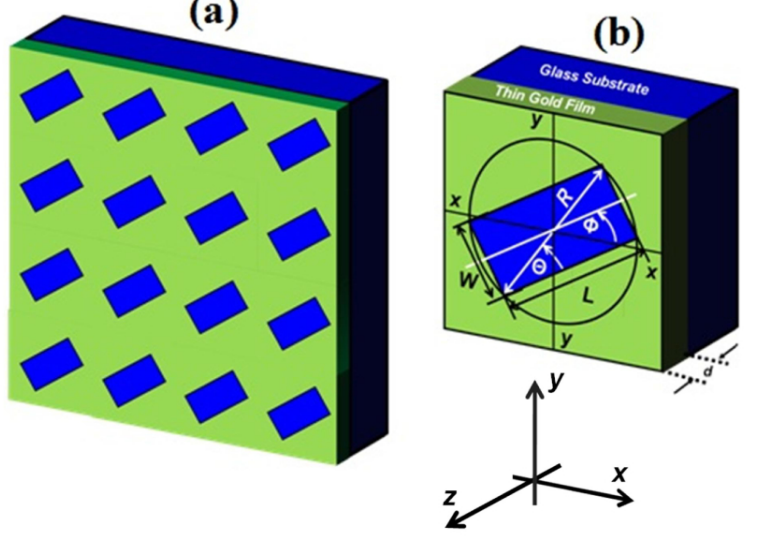

FIG. 1 (a) Layout of the designed plasmonic arrayed structure. (b) A unit cell of the designed structure.

shape and inscribed in circles of identical diameter. Figure 1(b) shows an enlarged unit cell that has a nano-hole of width $W$ and length $L$ determined by unequal scaling angle $\Theta$. Each rectangle nano-hole has an orientation angle $\Phi$ toward the $x$ direction, as shown in Figure 1(b).

During investigating the impact of rotating the rectangular nano-hole array on their transmission spectra, the structural periodicity and gold film thickness are kept constants at $425 \mathrm{~nm}$ and $200 \mathrm{~nm}$, respectively. The simulation domain size is $425 \mathrm{~nm} \times 425 \mathrm{~nm} \times 1000 \mathrm{~nm}$ cells in the Cartesian coordinates $x, y$, and $z$.

An absorbing boundary condition was rendered in the $z$ direction using anisotropic perfect matching layer, while, periodic boundary conditions were used in the $x$ and $y$ directions. The used refractive index value of the glass substrate is 1.5 and the relative permittivity $\varepsilon_{r}(\omega)$ of the dispersive gold film was determined using the Lorentz-Drude model as follows [15]:

$$
\varepsilon_{r}(\omega)=\varepsilon_{\infty}+\sum_{m=1}^{N} \frac{f_{m} \omega_{m}^{2}}{\omega_{o m}^{2}-\omega^{2}+i \omega \Gamma_{m}},
$$

where $\varepsilon_{\infty}$ denotes the permittivity at infinite frequency, $\mathrm{fm}$ is the oscillator strengths, and $\Gamma_{m}$ is the damping coefficient. The incident wave frequency and the resonant frequencies are represented by $\omega$ and $\omega_{\text {om }}$, respectively.

In order to realize a broadband simulation on the dispersive gold film, Gaussian modulated electromagnetic plane wave source was used. The continuous waves are centered at $680 \mathrm{~nm}$, linearly polarized in $y$-direction, and convolved with a Gaussian envelope function. In time domain, the light pulse is adjusted to have a half width of $0.8 \times 10^{-14} \mathrm{~s}$ and an offset time of $0.1 \times 10^{-14} \mathrm{~s}$. The simulation is performed at normal incidence (z-direction) of the plane wave through the nanohole arrays. The mesh size is adjusted to be small enough to catch the wave attenuation within the skin depth. As a result, the calculation mesh resolution is set as $5 \mathrm{~nm}(<0.1 \lambda)$ to get accurate results. The simulation is executed through 12,000 time step for a calculation time of $100 \mathrm{fs}$. An $x-y$ observation area is used to calculate the transmission spectral analysis at $400 \mathrm{~nm}$ away from the air / gold film interface.

\section{OPTICAL TRANSMISSION SPECTRAL RESPONSES OF THE REPORTED STRUCTURE}

In this section, we investigate the transmission spectra of the designed plasmonic structures using the FDTD method. The effect of varying different key parameters including the unequal scaling angle $\Theta$ and the orientation angle $\Phi$ on the optical transmission properties are discussed in the following subsections. At the end of this section, the impact of changing the substrate refractive index was studied for refractive index sensor at near-infrared wavelengths. By extension, the surface plasmonic resonance sensing was presented as an application for the reported nano-hole array.

\subsection{Different unequal scaling angle}

In this subsection, we investigate modifying the transmission spectra of the gold-perforated rectangular nano-hole arrays by changing the unequal scaling angle $\Theta$ within a range of $2.5^{\circ}$ $87.5^{\circ}$. During this study, the lattice constant, gold film thickness, substrate refractive index, and orientation angle $\Phi$ are kept constants at $425 \mathrm{~nm}, 200 \mathrm{~nm}, 1.5$, and $0^{\circ}$, respectively.

Figure 2 shows the transmission spectra of four different rectangular nano-hole arrays of various unequal scaling angles $\left(\Theta=10^{\circ}, 25^{\circ}, 45^{\circ}\right.$, and $\left.55^{\circ}\right)$. For the purpose of comparison, the spectrum of a reference circular nano-hole array sample circumscribed about those four rectangular arrays, but without the presence of the rectangles, is also shown in Figure 2. In this figure, the notations "P1" and "P2" are used to denote two transmission groups of resonance peaks. The first group "P1" is located at wavelengths larger than $0.68 \mu \mathrm{m}$, while, "P2" is located at wavelengths less than $0.68 \mu \mathrm{m}$. Transmission peaks occur due to matching between the surface plasmon polaritons (SPP) excited on the gold surfaces and the nano-hole array structural periodicity. At normal incidence, the SPP resonance wavelengths $\lambda_{S P P}$ of nano-hole array of square lattice constant can be approximated by the following equation [15]:

$$
\lambda_{S S P}(i, j)=\operatorname{Re}\left[\frac{P}{\sqrt{i^{2}+j^{2}}} \sqrt{\frac{\varepsilon_{d} \varepsilon_{m}}{\varepsilon_{d}+\varepsilon_{m}}}\right],
$$

where $\varepsilon_{d}$ and $\varepsilon_{m}$ respectively represent the relative permitivities of the adjacent dielectric medium and the metal, $(i, j)$ are integers, and $P$ represents the lattice constant.

Using Eq. (2), the sub-wavelength array with square nanohole $\left(\Theta=45^{\circ}\right)$, for example, has resonance wavelengths of $0.64 \mu \mathrm{m}$ and $0.75 \mu \mathrm{m}$ that can be assigned to $\lambda_{S P P}(1,1)$ of the air / gold "P2" and $\lambda_{S P P}(1,0)$ of the gold / substrate "P1" interfaces, respectively.

Localized surface plasmons (LSP) are formed within the nanohole with lateral dimensions in the range of the penetration depth. The cut-off wavelength $\lambda_{C}$ depends on the physical dimension of the nano-hole and may be extended by penetration of the field into the metal along the $x$-direction [19]. 


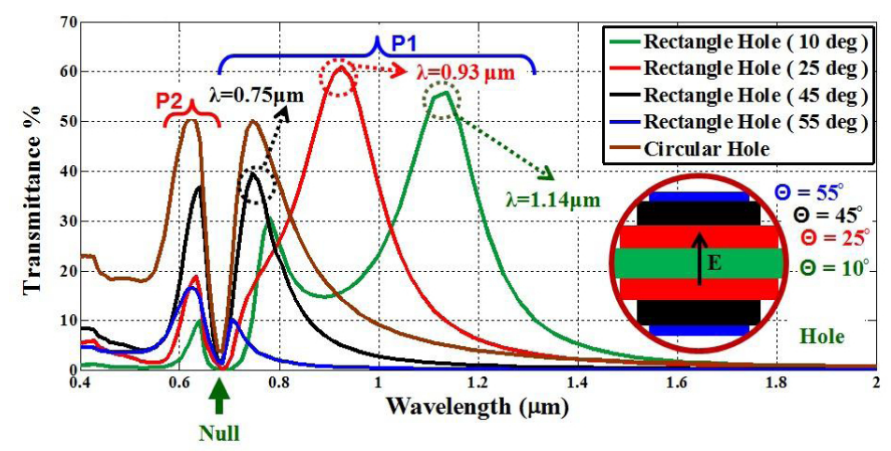

FIG. 2 Transmission spectra of gold thin film perforated with rectangular holes at different $\Theta$ values. The brown solid curve represents the transmission spectrum of the reference circular nano-holes sample.

As shown in Figure 2, the cut-off wavelengths of the circular nano-hole array $\left(\lambda_{C} \approx 1.8 \mu \mathrm{m}\right)$ is larger than that of the square nano-hole array $\left(\lambda_{C} \approx 1.25 \mu \mathrm{m}\right)$. The ratio of the cutoff wavelength of the circular nano-hole array to that of the square array is identical to the ratio of the diameter of the circle to the square side.

An even larger increase in the cut-off condition is found by considering coupled SP waves along the $y$-direction. For example, the transmission spectrum of the subwavelength rectangular array with $10^{\circ}$ unequal scaling angle shows interesting asymmetrical features. It exhibits one peak centered at wavelength of $0.64 \mu \mathrm{m}$, belonged to "P2", and shows two splitting peaks at $0.77 \mu \mathrm{m}$, and $1.14 \mu \mathrm{m}$, belonged to "P1".

Comparing the spectra of the subwavelength rectangular hole structures, one can observe that the location of the null transmission and peaks located on its short-wavelength side are independent of hole shape and dimensions. These spectral features depend only on the periodicity of the array and the refractive index of both the film and the surrounding media $[9,10]$. However, as shown in Figure 2, the peaks of the spectra located on the long-wavelength side of the null transmission are hole-geometry dependent.

Figure 3 shows an unequal scaling peak chart that indicates the locations and intensities of the transmission spectra peaks of rectangular nano-hole arrays with different dimensions. The results of Figure 3 show that as the unequal scaling angle $\Theta$ increases from $0^{\circ}$ to about $20^{\circ}$, the positions of the two splitting peaks of "P1", indicated by white and black stars, are dramatically blue shifted. With further increase in $\Theta$, the splitting peaks of "P1", white stars, merge and continue shifting to shorter wavelengths till the transmission is significantly suppressed below the dashed white line at $\Theta=65^{\circ}$.

Transmission suppression mainly depends on the rectangle side $L$ which is perpendicular to the direction of the electric field. For $\Theta \geq 65^{\circ}, L$ is relatively small and the cut-off occurs at a short wavelength which yields to transmission suppression. For $\Theta \leq 65^{\circ}$, the physical dimension of $L$ becomes larger and higher cut-off wavelengths are achieved which results in the appearance of the resonance peaks. For small values of $\Theta, \Theta<20^{\circ}$, the short rectangle width $W$ increases the

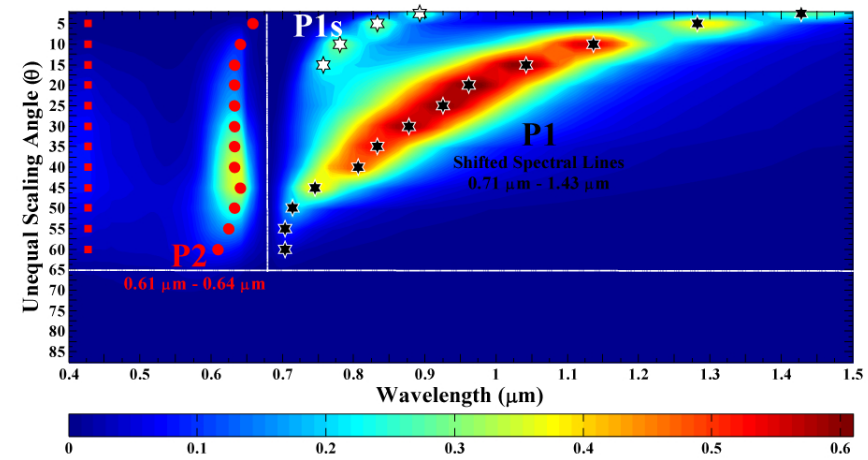

FIG. 3 The unequal scaling peak chart. " $\mathrm{P}_{1}$ " and " $\mathrm{P}_{2}$ " are used to define the two transmission groups of resonance peaks and $\mathrm{P}_{1} \mathrm{~s}$ denotes the splitted peaks from the "P1" group.
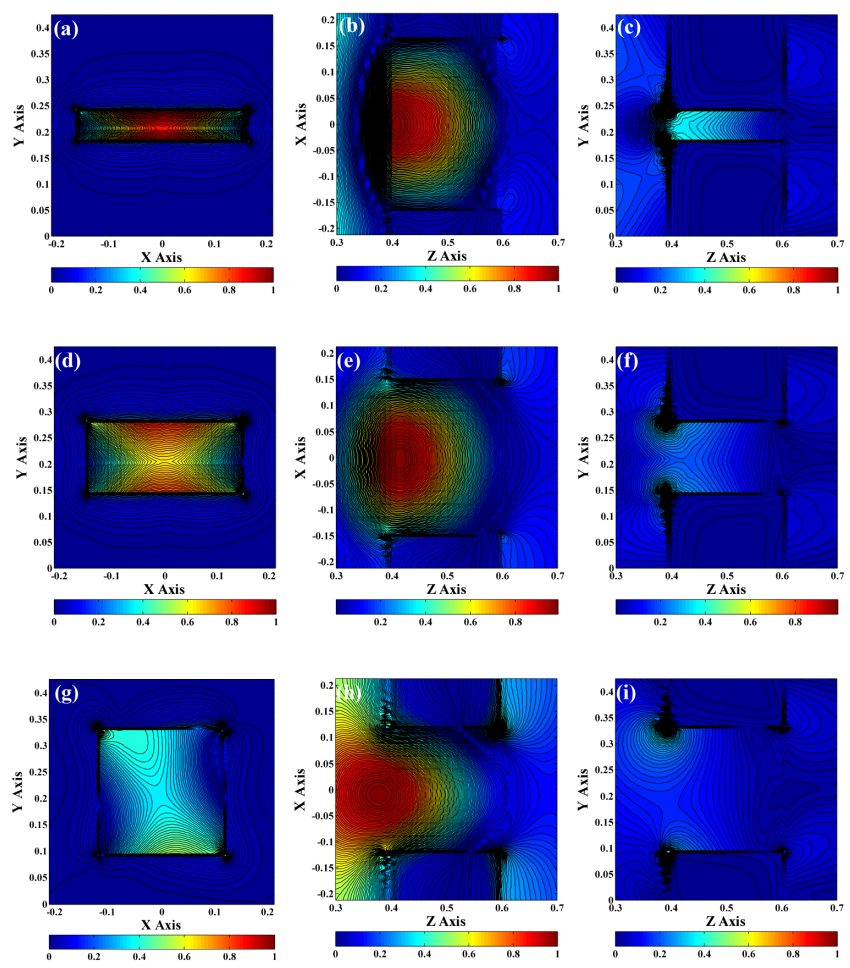

FIG. 4 The total electric field in the three principal Cartesian planes at the middle of a nano-hole when (a), (b), and (c) $\Theta=10^{\circ},(d),(e)$, and (f) $\Theta=25^{\circ}$, and (g), (h), and (i) $\Theta=45^{\circ}$.

SPs coupling between the long edges of the nano-hole. The formed LSP resonances combine with SPP which results in the observed blueshift of the resonance wavelengths and peaks splitting. The red circles illustrate that the resonance positions of "P2" are independent of the unequal scaling angle $\Theta$.

In order to validate our observations, the total electric field in the three principal Cartesian planes at the middle of a nanohole is demonstrated in Figure 4. The field distributions were calculated for the selected nano-hole arrays $\left(\Theta=10^{\circ}, 25^{\circ}\right.$, and $\left.45^{\circ}\right)$ at $0.68 \mu \mathrm{m}$ wavelength. It gives a clear picture of the interplay between SPP and LSP.

Distributions on $y-z$ planes, shown in Figures 4(c), (f), and (i), are stronger near the metal/substrate interface. This result is 
consistent with that obtained in Figure 2 that the peak transmittance of P1 is always higher than that of P2. Field distributions in x-y plane, illustrated in Figures 4(a), (d), and (g), indicate strong field is accumulating on the surface of the nanohole at upper and lower sides $(L)$. In addition, they show LSP formation as a result of decreasing L. LSP is responsible for peak splitting shown in Figure 2. This result is consistent with the electric field distributions in $x$-z plane. Figure 4 (b) shows LSP collect more optical energy to funnel to the other side of the metal surface.

\subsection{Rotation of the array}

As demonstrated in the previous section, the plasmonic resonance wavelengths can be modified by varying the nano-hole dimensions through the unequal scaling angle $\Theta$. In this subsection, we investigate the impact of changing the orientation angle $\Phi$ on the properties of the transmission spectrum. During this study, $\Phi$ changes within a range of $0^{\circ}-180^{\circ}$ with $5^{\circ}$ step for three aperture arrays of different geometries $\left(\Theta=10^{\circ}\right.$, $25^{\circ}$, and $45^{\circ}$ ). In addition, the lattice constant, gold film thickness, and substrate refractive index are respectively kept constants at $425 \mathrm{~nm}, 200 \mathrm{~nm}$, and 1.5 and the electric field remains in the $y$-direction.

Figure 5 shows the impact of changing $\Phi$ on the transmission spectra of the selected three rectangular nano-hole arrays. Unequal scaling angles, hole shape, and dimensions are presented in the panels.

Using the results shown in Figures 5(a) and (b), one can conclude that the amplitudes of the transmission spectra of the asymmetrical rectangle apertures $\left(\Theta=10^{\circ}\right.$ and $\left.25^{\circ}\right)$ are strongly modulated by the orientation angle. Starting form $\Phi$ $=0^{\circ}$, as the orientation angle increases, the transmission peaks intensities decrease till it nearly reach zero at $\Phi=90^{\circ}$. With further increasing, the transmission peaks intensities increase again. In other words, the transmission intensity shows a 2fold symmetry around $\Phi=90^{\circ}$.

In contrast, from Figure 5(c) of the symmetrical square aperture $\left(\Theta=45^{\circ}\right)$, the intensity of the spectrum at the resonance wavelengths of "P1" shows less orientation dependencies. By increasing the orientation angle $\Phi$ up to $45^{\circ}$ the resonance wavelengths of "P1" shows a redshift from $0.75 \mu \mathrm{m}$ to $0.79 \mu \mathrm{m}$. With further increasing, the positions of the enhanced peaks display a reverse blueshift which results in 4fold symmetry around $\Phi=45^{\circ}, 90^{\circ}$, and $135^{\circ}$.

Figure 6 demonstrates the orientation angle dependencies of transmission peaks intensities at the resonance wavelengths indicated in the panels at $\Theta=10^{\circ}, 25^{\circ}$, and $45^{\circ}$. It is clear in the case of the asymmetric rectangle nano-hole arrays, Figure 6(a) and (b), that the peak intensities are significantly modified by changing the orientation angle. Inspired by the polarizer, the red solid lines fit the peak transmittance $T_{p}(\Phi)$ in Figures (a) and (b) according to the relation

$$
T_{P}(\Phi)=k\left[\begin{array}{c}
\cos ^{2}\left(\Phi-\Phi_{o}\right)+ \\
\cos \left(\Phi-\Phi_{o}\right) \sin \left(\Phi-\Phi_{o}\right)
\end{array}\right],
$$

where $\Phi_{o}$ and $k$ are fitting parameters. It is found that the
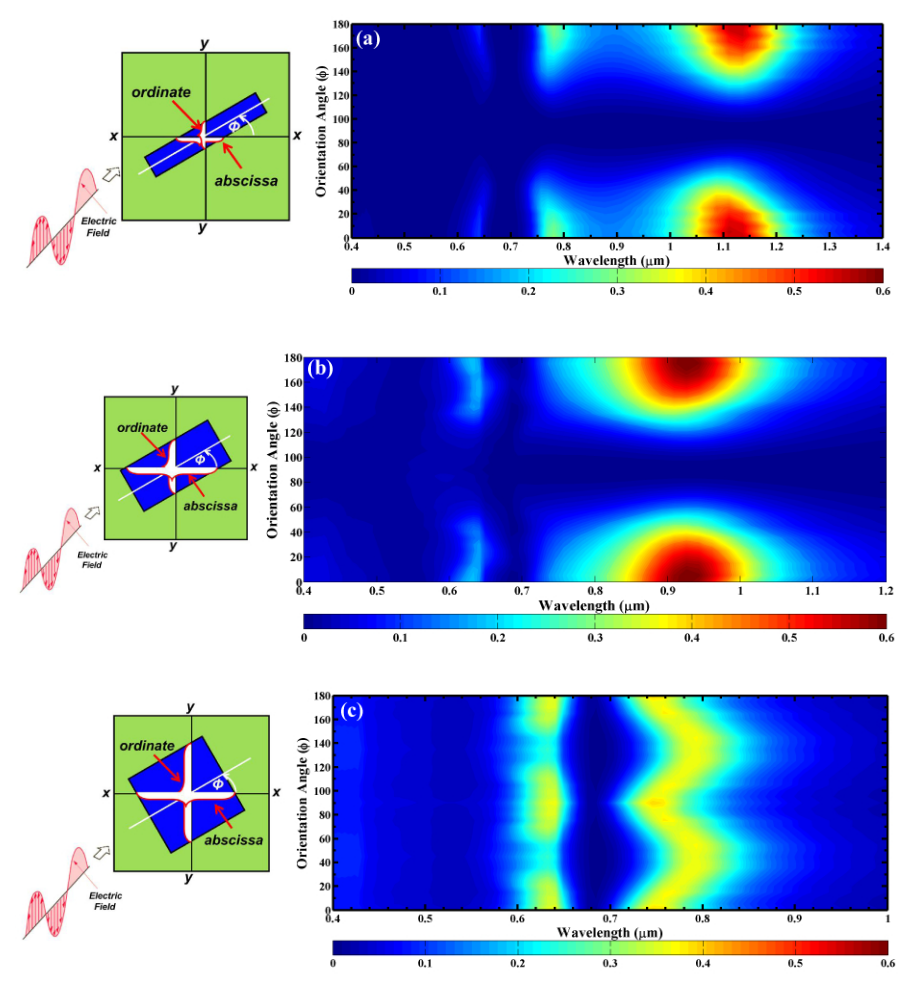

FIG. 5 Impact of changing $\Phi$ on the transmission spectra of the selected three rectangular nano-hole arrays. (a) $\Theta=10^{\circ}$, (b) $\Theta=25^{\circ}$, and (c) $\Theta=45^{\circ}$.

phase shift fitting parameter $\Phi_{o}$ is independent of $\Theta$ and has the same value $\left(\Phi_{o}=20^{\circ}\right)$ for the two shown rectangle nanohole arrays. However, the value of the amplitude fitting parameter $k$ changes with $\Theta$.

Figure 6(c) shows that the square nano-holes have less orientation dependencies. The spectrum amplitudes at the resonance wavelength of "P1" are almost constant ( $\approx 36 \%-39 \%)$. The electron oscillations are formed on the surface perpendicular to the direction of the electric field cause SPP. The LSP is enhanced with decreasing the lateral distance (side parallel to the electric field) in the range of the skin depth. This means that abscissa plays the main role in the cut-off and defines the resonance peak intensity, while the ordinate is responsible for the resonance wavelength shift. For $\Theta=10^{\circ}$ and $0^{\circ}<\Phi<60^{\circ}$, the abscissa is dramatically changing while the ordinate is almost constant. As a result, the shape of transmission curve is just decaying with no change in the peaks positions. For $\Phi>60^{\circ}$, the abscissa is relatively small which causes the cutoff. For the square array, both the abscissa and the ordinate are changing over the whole range of $\Phi$ which results in changing of peaks positions.

It should be noted that, the orientation angle determines the polarization angle between incident polarization and the rectangle structure. If the orientation angle is fixed, similar results can be obtained by adjusting the incident polarization. Finally, we investigate the influence of the oblique incidence light on the transmission spectra of the gold-perforated rectangular nano-hole array $\left(\Theta=25^{\circ}\right)$. As illustrated in Figure 7, the structure is durable to the oblique incidence. With the increasing of the incident angle, the resonance wavelength does not change while the transmission peak shows some degrada- 
(a)

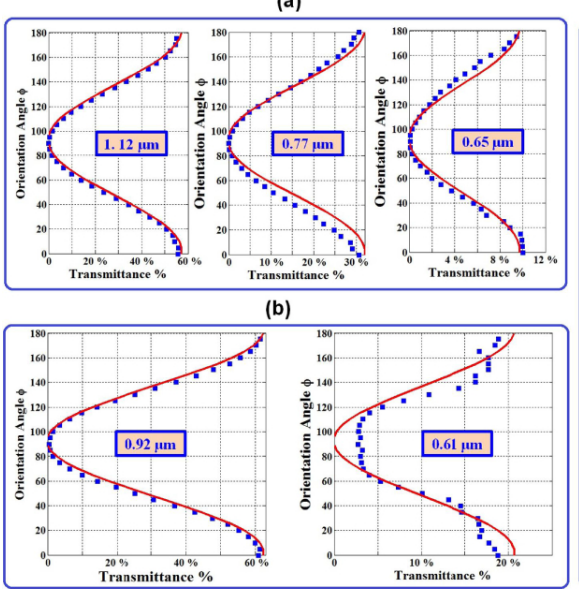

(c)

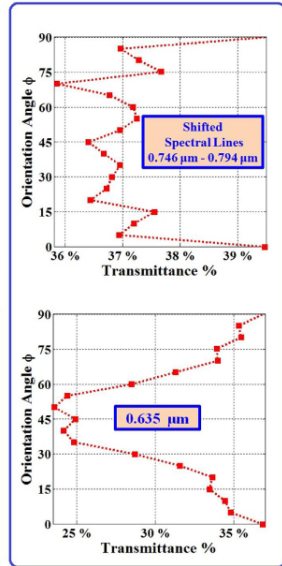

FIG. 6 Orientation angle dependencies of transmission peaks intensities at the wavelengths indicated in panels when (a) $\Theta=10^{\circ}, L=322 \mathrm{~nm}$, and $W=57 \mathrm{~nm}$, (b) $\Theta=25^{\circ}, L=296 \mathrm{~nm}$, and $W=138 \mathrm{~nm}$, and $(c) \Theta=45^{\circ}$ and $L=W=231 \mathrm{~nm}$.

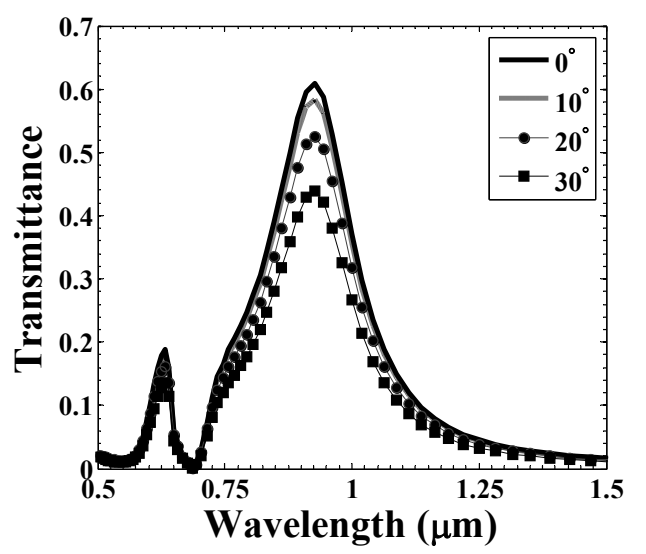

FIG. 7 Impact of the incident angle on the transmission spectra.

tion. The transmittance remains about $70 \%$ from its peak with 30 degree incident angle.

\subsection{Nano-hole array as refractive index sensor}

We here investigate the effect of changing the refractive index of the substrate in the range of $1.3-1.6$ on both the transmission and absorption spectra of the gold-perforated rectangular nano-hole array $\left(\Theta=25^{\circ}\right)$. Figures $8(\mathrm{a})$ and $(\mathrm{b})$ exhibit the transmission and absorption spectra, respectively, at different values of the refractive index of the substrate. As can be seen from these figures, the resonance wavelengths show a linear redshift with nearly constant slope with increasing the substrate refractive index. In particular, the refractive index sensitivities $S_{n}=\delta \lambda_{S P} / \delta$ n of the designed structures at the resonance wavelengths are 282.1 and $219.5 \mathrm{~nm} / \mathrm{RIU}$ when transmission and absorption spectra are considered, respectively.
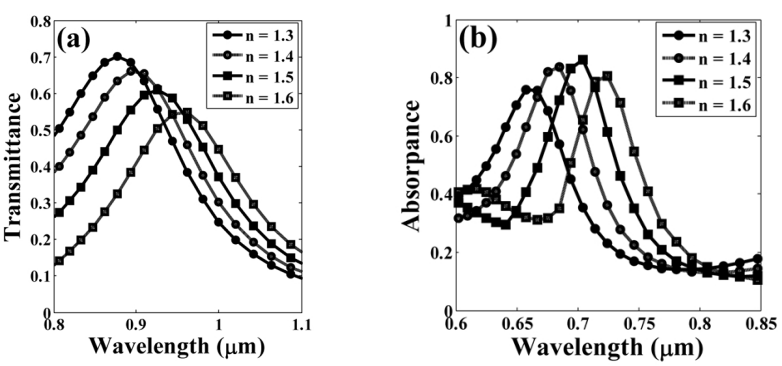

FIG. 8 The impact of changing the substrate refractive index on the optical (a) transmission (b) absorption spectra of the gold-perforated rectangular nano-hole arrays.

\section{CONCLUSIONS}

In this work, we comprehensively investigate the EOT properties of the rectangular plasmonic nano-hole arrays. We find that changing the dimensions of the rectangular nano-holes can significantly tune the resonance wavelengths located at values larger than $0.68 \mu \mathrm{m}$. Additionally, the nano-hole dimensions can also control the peak intensity values at wavelength less than $0.68 \mu \mathrm{m}$. It is also observed that rotating the rectangular nano-hole array around the $x$-axis causes significant change in the peak intensity values of the transmission spectra. However, it has a less impact on the peak intensity values of the square nano-hole arrays.

\section{References}

[1] F. Fida, L. Varin, S. Badilescu, M. Kahrizi, and V. Truong, "Cold nanoparticle ring and hole structures for sensing proteins and antigen-antibody interactions," Plasmonics 4, 201-209 (2009).

[2] X. Dang, J. Qi, M. T. Klug, P. Chen, D. S. Yun, N. X. Fang, P. T. Hammond, and A. M. Belcher, "Tunable localized surface plasmon-enabled broadband light-harvesting enhancement for high-efficiency panchromatic dye-sensitized solar cells," Nano Lett. 13, 637-642 (2013).

[3] M. Yi, D. Zhang, X. Wen, Q. Fu, P. Wang, Y. Lu, and H. Ming, "Fluorescence enhancement caused by plasmonics coupling between silver nano-cubes and silver film," Plasmonics 6, 213-217 (2011).

[4] I. Ashry, B. Zhang, S. V. Stoianov, C. Daengngam, J. R. Heflin, H. D. Robinson, and Y. Xu, "Probing the photonic density of states using layer-by-layer self-assembly," Opt. Lett. 37, 1835-1837 (2012).

[5] Q. Wang, X. Wang, X. Li, and S. Wu, “Transmission control property of a nano-optical system made by an antenna over a Bowtie aperture," Plasmonics 8, 1141-1146 (2013).

[6] T. W. Ebbesen, H. J. Lezec, H. F. Chaemi, T. Thio, and P. A. Wolff, "Extraordinary optical transmission through sub-wavelength hole arrays," Nature 391, 667-669 (1998).

[7] H. Ghaemi, T. Thio, D. Grupp, T. Ebbesen, and H. Lezec, "Surface plasmons enhance optical transmission through subwavelength holes," Phys. Rev. B 58, 6779-6782 (1998).

[8] S. Orbons, M. Haftel, C. Schlockermann, D. Freeman, M. Milicevic, T. Davis, B. Davies, D. Jamieson, and A. Roberts, "Dual resonance mechanisms facilitating enhanced optical transmission in coaxial waveguide arrays," Opt. Lett. 33, 821-823 (2008). 
[9] A. K. Azad, Y. Zhao, W. Zhang, and M. He, "Effect of dielectric properties of metals on terahertz transmission subwavelength hole arrays," Opt. Lett. 31, 2637-2639 (2006).

[10] M. Kofke, D. Waldeck, Z. Fakhraai, S. Ip, and G. Walker, "The effect of periodicity on the extraordinary optical transmission of annular aperture arrays," Appl. Phys. Lett. 94, 023104 (2009).

[11] L. Lin, and A. Roberts, "Light transmission through nanostructured metallic films: coupling between surface waves and localized resonances," Opt. Express 19, 2626-2633 (2011).

[12] Y. Wang, Y. Qin, and Z. Zhang, "Extraordinary optical transmission property of $x$-shaped plasmonic nanohole arrays," Plasmonics 9, 203-207 (2014).

[13] R. Biswas, S. Neginhal, C. Ding, I. Puscasu, and E. Johnson, “Mechanisms underlying extraordinary transmission enhancement in subwavelength hole arrays," J. Opt. Soc. Am. B 24, 2589-2596 (2007).

[14] T. Vallius, J. Turunen, M. Mansuripur, and S. Honkanen, “Transmission through single subwavelength apertures in thin metal films and effects of surface plasmons," J. Opt. Soc. Am. A 21, 456-463 (2004).
[15] M. Irannejad, and B. Cui, "Effects of refractive index variations on the optical transmittance spectral properties of the nano-hole arrays," Plasmonics 8, 1245-1251 (2013).

[16] Battula, S. Chen, Y. Lu, R. Kniz, and K. Reinhardt "Tuning the extraordinary optical transmission through subwavelength hole array by applying a magnetic field," Opt. Lett. 32, 2692-2694 (2007).

[17] G. C. des Francs, D. Molenda, U. C. Fischer, and A. Naber, "Enhanced light confinement in a triangular aperture: experimental evidence and numerical calculations," Phys. Rev. B 72, 165111 (2005).

[18] R. Wannemacher, "Plasmon-supported transmission of light through nanometric holes in metallic thin films," Opt. Commun. 195, 107-118 (2001).

[19] R. Gordon, and A. Brolo, "Increased cut-off wavelength for a subwavelength hole in a real metal," Opt. Express 13, 1933-1938 (2005). 